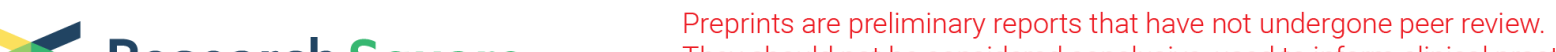 Research Square They should not be considered conclusive, used to inform clinical practice, or referenced by the media as validated information.
}

\section{An integrated omics analysis reveals the gene expression profiles of rapeseed, castor bean, and maize for seed oil biosynthesis}

Nian Liu

Oil Crops Research Institute Chinese Academy of Agricultural Sciences https://orcid.org/0000-00031325-0087

Jing Liu

Oil Crops Research Institute Chinese Academy of Agricultural Sciences

Shihang Fan

Oil Crops Research Institute Chinese Academy of Agricultural Sciences

Hongfang Liu

Oil Crops Research Institute Chinese Academy of Agricultural Sciences

Xue-Rong Zhou

CSIRO Agriculture and Food

Wei Hua

Oil Crops Research Institute Chinese Academy of Agricultural Sciences

Ming Zheng ( $\nabla$ zhengming@caas.cn)

Oil Crops Research Institute Chinese Academy of Agricultural Sciences https://orcid.org/0000-00016757-7531

\section{Research Article}

Keywords: seed oil biosynthesis, rapeseed, castor bean, maize, transcriptome analyses, lipid-related genes, carbohydrate-related genes

Posted Date: December 3rd, 2021

DOI: https://doi.org/10.21203/rs.3.rs-1129584/v1

License: (a) (1) This work is licensed under a Creative Commons Attribution 4.0 International License.

Read Full License 


\section{Abstract \\ Background}

Seed storage lipids are valuable for human diet and for the sustainable development of mankind. In recent decades, many lipid metabolism genes and pathways have been identified, but the molecular mechanisms that underlie species differences in seed oil biosynthesis are not fully understood.

\section{Results}

To investigate the molecular mechanisms of seed oil accumulation in different species, we performed comparative genome and transcriptome analyses of rapeseed and castor bean, which have high seed oil contents, and maize, which has a low seed oil content. The results uncovered the molecular mechanism of the low and high seed oil content in maize and castor bean, respectively. Transcriptome analyses showed that more than $61 \%$ of the lipid- and carbohydrate-related genes were regulated in rapeseed and castor bean, but only $20.1 \%$ of the lipid-related genes and $22.5 \%$ of the carbohydrate-related genes were regulated in maize. Compared to rapeseed and castor bean, fewer lipid biosynthesis genes but more lipid metabolism genes were regulated in the maize embryo. More importantly, most maize genes encoding lipid-related transcription factors, triacylglycerol (TAG) biosynthetic enzymes, pentose phosphate pathway (PPP) and Calvin Cycle proteins were not regulated during seed oil synthesis, despite the presence of many homologs in the maize genome. These results revealed the molecular underpinnings of the low seed oil content in maize. In castor bean, we observed differential regulation of vital oil biosynthetic enzymes and extremely high expression levels of oil biosynthetic genes, which were consistent with the rapid accumulation of oil in castor bean developing seeds.

\section{Conclusions}

Compared to oil seed (rapeseed and castor bean), less oil biosynthetic genes were regulated during the seed development in non-oil seed (maize). These results shed light on molecular mechanisms of lipid biosynthesis in rapeseed, castor bean, and maize. They can provide information on key target genes that may be useful for future experimental manipulation of oil production in oilseed crops.

\section{Background}

Plant storage lipids are renewable and economically valuable resources; they are important for human diet and for use as biodiesel [1, 2]. There is a pressing need for enhancing the seed oil content (SOC) of oil crops to meet the sharply increasing demand for plant oil consumption. Lipid biosynthesis is a complex biological process which is precisely regulated by multiple genes [3]. In general, fatty acids (FAs) are de novo synthesized in the plastid from acetyl-coenzyme A (acetyl-CoA) precursors, condensing into long-chain acyls by numerous enzymes such as acetyl-CoA carboxylase (ACCase) [4-6], malonyl- 
CoA:ACP malonyltransferase (MCMT), $\beta$-ketoacyl-ACP synthase (KAS) complex [7, 8], hydroxyacyl-ACP dehydrase (HAD), enoyl-ACP reductase (ENR), $\beta$-ketoacyl-ACP reductase (KAR) [9], and acyl carrier protein (ACP). The products of FA sysnthesized with chain length of $\mathrm{C} 16$ or $\mathrm{C} 18$ can be unsaturated by stearoylACP desaturase (SAD). Both saturated and unsaturated FAs are released from acyl-ACP by acyl-ACP thioesterase (FAT) [10] and exported to cytosol. The resulting FAs pools are then transported to the endoplasmic reticulum (ER) for triacylglycerol (TAG) assembly by enzymes such as glycerol 3-phosphate acyltransferase (GPAT) [11, 12], lysophosphatidic acid acyltransferase (LPAAT) [13], phosphatidic acid phosphatase (PAP) [14], diacylglycerol acyltransferase (DGAT) [9, 15-17], and phospholipid:diacylglycerol acyltransferase (PDAT) $[18,19]$. Finally, the mature TAGs are transferred and stored in subcellular structures called oil bodies or oleosomes. Oleosin, caleosin, and steroleosin have been shown to be indispensable for oil body formation and can regulate $\mathrm{SOC}[20,3]$. Many transcription factors (TFs) also participate in seed development and nutrient accumulation. TFs such as WRINKLED1 (WRI1), LEAFY COTYLEDON1/2 (LEC1/2), ABSCISIC ACID INSENSITIVE3 (ABI3), FUSCA3 (FUS3), PICKLE (PKL), and VP1/ABI3-LIKE (VAL) have been proved to regulate SOC in species such as Arabidopsis, rapeseed, soybean, castor bean, and others [14, 21-24].

Lipid biosynthesis requires substrates and energy obtained from carbon metabolism. There is strong evidence showing that glycolysis provides acetyl-CoA, dihydroxyacetone phosphate, ATP, and NADH for lipid biosynthesis [25-27], whereas the pentose phosphate pathway (PPP) mainly produces the reductant NAPDH that is needed for fatty acid synthesis [27]. It has been shown that many carbohydrate-related genes impact lipid accumulation. For example, pyruvate kinase (PK) catalyzes the production of pyruvate and ATP in glycolysis, and the loss of function of $P K$ in Arabidopsis drastically reduced FA content [28, 29]. Phosphoenolpyruvate carboxylase (PEPC) catalyzes the production of oxaloacetate (OAA) from phosphoenolpyruvate (PEP), and RNA interference of PEPC increased the oil content of cotton [30].

For decades, studies of seed oil biosynthesis have been focused mainly on comparisons within species [31-37]. With the rapid development of sequencing technologies in recent years, several comparative transcriptome analyses have been performed to explore the molecular mechanisms that underlie differences in seed oil biosynthesis between different species. The transcriptomes of four developing oilseeds (Ricinus communis, Brassica napus, Euonymus alatus, and Tropaeolum majus) were sequenced based on deep expressed sequence tags (ESTs) and some conserved lipid-related modules were found [38]. Comparative genomic analyses were conducted on three low-oil grasses (Sorghum bicolor, Setaria italica, and Oryza sativa) and four high-oil dicots (Glycine max, Gossypium raimondii, R. communis, and Arabidopsis thaliana) to investigate the mechanisms of their differences in seed oil accumulation [39]. Zhang et al. [40] performed an integrated omics analysis to investigate acyl lipid metabolism genes and carbon metabolism genes in soybean, rapeseed, Arabidopsis, and sesame seeds. To date, inter-species comparative transcriptome analyses were carried out among several species, but the molecular mechanism of differential oil biosynthesis between maize (which is one of the most important worldwide food crops and vital source of vegetable oil) and oil seeds (such as rape and castor bean) remains obscure. 
In addition, different plant species store their lipids in different organs or tissues, therefore, it is important to understand the mechanism difference in oil accumulation from different tissues. Most plants store lipids in the embryo [41, 42], but some exalbuminous seeds such as castor bean and Jatropha curcas store lipids in the endosperm $[14,43,8]$. Additionally, plants that have developed pericarps, such as the oil palm, can accumulate lipids in the mesocarp [44]. To date, the molecular mechanisms that underlie differences in lipid accumulation among different plant species remain unclear. Comparisons between species have focused mainly on whole seeds, but differences in seed oil biosynthesis at the level of the specific lipid storage tissues have not been addressed.

This study is to investigate the molecular mechanisms responsible for differences in lipid accumulation in the storage organs of high- and low-oil-content species. Embryos of rapeseed ( $B$. napus) and maize (Zea mays) and endosperm tissues of castor bean ( $R$. communis) were collected from the developing seeds at different developmental stages for transcriptome analyses. Although the gene copy numbers for the individual genes are different among these three species, clear difference in regulation of lipid biosynthetic pathways was observed. Higher number of the lipid- and carbohydrate-related genes were regulated in rapeseed and castor bean than in maize. The expression levels of oil biosynthetic genes were highest in castor bean.

\section{Results}

\section{Copy number variation in lipid- and carbohydrate-related genes of rapeseed, castor bean, and maize}

To eliminate the effect of species ploidy, the relative copy numbers of key regulators that participate in seed oil biosynthesis were analyzed in these three species. Copy numbers of genes that encoding proteins involving in FA biosynthesis, TAG assembly, and oil body formation were higher in rapeseed than in castor bean and maize (Fig. 1a). This result probably reflects the fact that rapeseed is an allopolyploid and contains duplicates of most genes [45]. All lipid biosynthesis genes had more copies in maize than in castor bean except for ACCase, the key rate-limiting enzyme of FA biosynthesis (Fig. 1a). It has been wellknown that ACCase contains the homomeric ACC2 and heteromeric ACCase complex composed of CT-a, $C T-\beta, B C C P$, and $B C$ subunits. Our findings revealed that the castor bean and rapeseed genomes contained the heteromeric ACCase but not the homomeric $A C C 2$, whereas the maize genome contained only homomeric ACC2 (Fig. 1a). In addition to different copy numbers of lipid biosynthesis genes, the three species also had different copy numbers of genes encoding oil biosynthesis regulators. All lipid-related TFs had fewer copy numbers in castor bean than in rapeseed (Fig. 1a). The relative copy numbers of lipid-related TF genes in maize were inconsistent: maize had the greatest numbers of $W R / 1, A B / 3$, and VAL2 genes among three species, but the lowest copy numbers of LEC1 and FUS3. More importantly, $L E C 2$ and $P K L$ genes were not present in the maize genome (Fig. 1a). In addition, rapeseed had the highest copy numbers of carbohydrate-related genes that functioned in sucrose metabolism, glycolysis, the PPP, and the Calvin Cycle, followed by maize and castor bean (Fig. 1b). These findings revealed that 
the copy numbers of most lipid- and carbohydrate-related genes were highest in rapeseed, lower in maize, and lowest in castor bean.

\section{Identification of DEGs during seed oil biosynthesis in rapeseed, castor bean, and maize}

Rapeseed embryo, castor bean endosperm, and maize embryo tissues were collected at four developmental stages (S1-S4) for SOC and transcriptome analysis (Fig. 2a). Seed oil content increased as seed development progressed and reached its highest level at S3 in rapeseed, castor bean, and maize (Fig. 2a). After transcriptome sequencing and data processing, relative gene expression levels at stages 2 , 3 , and 4 were compared to that at stage 1 to identify the differentially expressed genes (DEGs). In total, 47 408, 10 708, and 7144 genes were differentially expressed over the course of seed development in rapeseed, castor bean, and maize, respectively (Fig. 2b). Moreover, lipid- and carbohydrate-related genes accounted for $3.5 \%$ and $1.3 \%$ of all DEGs in the rapeseed embryo, and similar numbers were found in the endosperm of castor bean or the embryo of maize (Fig. 2b). In contrast, when the number of lipid- and carbohydrate-related DEGs was compared with the number of lipid- and carbohydrate-related genes in the entire genomes, $61.8 \%(937 / 1516)$ of the lipid-related genes and $68.0 \%(607 / 892)$ of the carbohydraterelated genes in rapeseed were regulated in embryo; $61.2 \%$ (224/366) of the lipid-related genes and $61.7 \%$ $(142 / 230)$ of the carbohydrate-related genes in castor bean were regulated in endosperm (Table 1$)$. However, only $20.1 \%(112 / 558)$ of the lipid-related genes and $22.5 \%(82 / 365)$ of the carbohydrate-related genes in maize were regulated in the embryo (Table 1). To further investigate the regulation of every homologous lipid- and carbohydrate-related gene in these species, we calculated a regulation ratio for each homologous gene by dividing the number of differentially expressed gene copies in a species by the total number of gene copies in the species. The comparison of the regulated ratio between three species showed that most genes had a higher regulation ratio in rapeseed and castor bean than in maize (Fig. S1). We therefore concluded that fewer genes involved in seed oil accumulation were regulated during seed development in maize.

Table 1

The number of total and differential expressed lipid-related and carbohydrate-related genes in rapeseed, castor bean, and maize.

\begin{tabular}{|lllll|}
\hline & $\begin{array}{l}\text { All lipid- } \\
\text { related } \\
\text { genes }\end{array}$ & $\begin{array}{l}\text { Differential expressed } \\
\text { lipid-related genes }\end{array}$ & $\begin{array}{l}\text { All } \\
\text { carbohydrate- } \\
\text { related } \\
\text { genes }\end{array}$ & $\begin{array}{l}\text { Differential expressed } \\
\text { carbohydrate-related } \\
\text { genes }\end{array}$ \\
\hline Rapeseed & 1516 & $937(61.8 \%)$ & 892 & $607(68.0 \%)$ \\
\hline Maize & 558 & $112(20.1 \%)$ & 365 & $82(22.5 \%)$ \\
\hline $\begin{array}{l}\text { Castor } \\
\text { bean }\end{array}$ & 366 & $224(61.2 \%)$ & 230 & $142(61.7 \%)$ \\
\hline
\end{tabular}

We next performed Gene Ontology (GO) analysis to identify differentially regulated pathways between three species during seed oil accumulation. GO terms enriched in the rapeseed DEGs were mainly related 
to photosynthesis, response to stresses such as cold, water deprivation and abscisic acid, and lipid metabolic processes (Data S1). Castor bean DEGs were mainly enriched in $\mathrm{GO}$ terms related to protein biosynthesis and modification, lipid metabolic processes, and DNA replication (Data S1). In contrast, maize DEGs were enriched in GO terms such as regulation of transcription, DNA-templated, and spermine biosynthetic process (Data S1). Thus, more lipid related genes were regulated in rapeseed and castor bean than in maize during seed development.

Next, homologous genes were identified in rapeseed, castor bean, and maize, and 2108 genes were found to be conserved among these species. Furthermore, there were 5040 dicot-specific genes (shared by rapeseed and castor bean) and 1256 embryo-specific genes (shared by rapeseed and maize) (Fig. 2c). Enriched GO terms in these 2108 conserved genes included acetyl-CoA biosynthetic process from pyruvate, long-chain fatty acid biosynthetic process, carbohydrate metabolic process, and acyl-CoA metabolic process (Data S1). Those 5040 dicot-specific genes were enriched in $\mathrm{GO}$ terms such as negative regulation of fatty acid biosynthetic process, lipid catabolic process, and lipid oxidation, while those 1265 embryo-specific genes were enriched in GO terms including response to salt stress, response to auxin, regulation of jasmonic acid-mediated signaling pathway, and ethylene biosynthetic process (Data S1). Therefore, we speculated that oil biosynthesis and carbon partitioning were differentially regulated between rapeseed, castor bean, and maize seeds and that fewer oil biosynthesis genes were regulated in maize than in rapeseed and castor bean.

\section{Differential expression of lipid-related genes in rapeseed, castor bean, and maize during seed development}

Subsequently, we focused on the gene expression profiles of lipid-related genes that have been reported to regulate lipid biosynthesis, and classified these genes into different categories based on lipid-related metabolic pathways [3]. Comparison of lipid-related DEGs (LDEGs) between species revealed that fewer lipid biosynthesis genes such as plastidial fatty acid synthesis and TAG synthesis genes were regulated in maize than in rapeseed and castor bean (Fig. 3a). By contrast, more lipid metabolism genes such as those encoding cutin synthesis, aliphatic suberin synthesis, GDSL, galactolipid degradation, and lipase were regulated in maize (Fig. 3a). All DEGs in three species could be grouped into different clusters based on their gene expression patterns (Fig. S2). Nevertheless, rapeseed lipid biosynthesis genes were clustered into four expression profiles; these genes reached their highest expression levels at stage 2 (Fig. 3b). Likewise, maize and castor bean lipid biosynthesis genes were clustered into one and two expression profiles, respectively, and their highest expression levels were also appeared at stage 2 (Fig. 3b). Maize lipid metabolism genes were clustered into four expression profiles and showed increasing expression trends during seed development. By contrast, lipid metabolism genes in the four expression profiles of castor bean tended to decrease in expression (Fig. 3c). Genes in five out of eight rapeseed expression profiles also tended to decrease in expression during development, except profiles

34, 39 and 44, whose genes showed a temporary increase in expression at S2 or S3 (Fig. 3c). Thus, we concluded that fewer lipid biosynthesis genes but more lipid metabolism genes were regulated in the maize embryo during seed oil accumulation. 
More genes involved in FA biosynthesis and oil body formation were differentially expressed in rapeseed and castor bean than in maize (Fig. S3), even though these genes had higher copy numbers in maize than in castor bean (Fig. 1a). Interestingly, almost all key TAG biosynthesis enzymes (e.g., GPDH, GPAT9, DGAT, PDAT, and PDCT) showed no regulation during maize embryo development (Fig. 4a). Similarly, key TFs that participate in lipid biosynthesis (e.g., LEC1/2, ABI3, VAL1/2, ASIL1, and PKL) showed little regulation in the maize embryo (Fig. 4b). Therefore, we concluded that lack of regulation of lipid biosynthesis enzymes, especially key TAG assembly enzymes and lipid-related TFs, may lead to the low abundance of oil in maize embryo.

In addition to LDEG numbers, gene expression levels also differed between these species and may have led to differences in seed oil biosynthesis. There were significant differences in the fold changes of many lipid-related genes between rapeseed, castor bean, and maize. The key lipid biosynthesis regulator genes PKL and VAL2 showed opposite regulation patterns between rapeseed and castor bean at the four developmental stages, and they were not regulated in maize (Fig. 5a). The rate-limiting FA biosynthesis ACCase enzymes were up-regulated at S2 and S3 in rapeseed, but ACCase homologs were downregulated or not regulated in castor bean and maize at S2 and S3 (Fig. 5a). Similarly, numerous lipidrelated genes (e.g., FUS3, WRI1, LEC1/2, PDCH, GPDHC, and DGAT) were also differentially regulated in rapeseed, castor bean, and maize (Fig. S4). To compare the gene expression levels of lipid-related genes in three species, the average FPKM of all expressed genes was introduced to normalize the relative gene expression levels in each species. We found that most lipid-related genes had higher relative expression levels in castor bean than in rapeseed and maize (only homologous ACCase and DGAT genes were shown), a finding that might account for the rapid accumulation of oil in castor bean endosperm (Fig. 5b).

\section{Differential expression of carbohydrate-related genes in rapeseed, castor bean, and maize during seed development}

In addition to lipid-related genes, carbohydrate-related genes also play vital roles in seed oil biosynthesis. Comparison of carbohydrate-related metabolic pathways between species indicated that glycolysis, the PPP, and the Calvin Cycle were up-regulated in rapeseed and castor bean. Other carbohydrate metabolic pathways were up-regulated in castor bean and maize, while sucrose metabolism and sugar transport pathways were up-regulated in maize (Fig. 6a). The numbers of carbohydrate-related DEGs (CDEGs) associated with sugar transport, plastid transport, organic acids, the TCA, and other carbohydrate metabolic pathways were the highest in rapeseed, followed by castor bean and maize (Fig. S5).

Most enzymes that participate in the PPP and Calvin Cycle (e.g., 6-phosphogluconate dehydrogenase (6PGDH), ribulose-phosphate 3-epimerase (RPE), transketolase (TK), transaldolase (TA), ribulose bisphosphate carboxylase (RBCS), and phosphoribulokinase (PRK)) were not regulated in maize embryo. Numerous glycolytic enzymes (including key enzymes such as hexokinase (HXK), phosphofructokinase (PFK), phosphoglycerate kinase (PGK), and PK) were regulated only in the cytosol in maize embryo. By contrast, they were differentially expressed in the cytosol and plastid of rapeseed embryo and castor 
bean endosperm (Fig. 6b). More interestingly, invertase (INV) genes that play important roles in sucrose metabolism functioned only in the cytosol in castor bean endosperm. This differed from the situation in rapeseed and maize embryos, in which INV genes were differentially expressed in the cytosol, plastid, cell wall, and vacuole (Fig. 6b). Another vital sucrose metabolism enzyme, sucrose synthase (SuSy), plays important roles in carbon fixation and metabolism. We found that more SuSy homologs were regulated in castor bean and rapeseed than in maize: 9 of 14 SuSy genes were regulated in rapeseed, and all five SuSy genes were regulated in castor bean, but only 3 of 14 SuSy genes were regulated in maize (Fig. 2b and $6 \mathrm{~b})$. Analysis of gene expression patterns showed that most carbohydrate-related genes were highly expressed at S1 and S2 in castor bean and rapeseed, but were highly expressed at S2 and S3 in maize (Fig. 6c). We therefore speculated that reduced regulation of carbohydrate-related genes may limit the supply of substrates for oil biosynthesis in maize.

\section{Discussion}

Thousands of genes were regulated during seed development, underscoring the sophisticated coordination of seed development and storage accumulation in plants. Plant seed oil biosynthesis is regulated by lipid- and carbohydrate-related genes [3]. Our research showed that most of these genes had the highest copy numbers in rapeseed, which is consistent with its allopolyploid genome in which most genes are duplicated and have redundant functions [45]. Most lipid- and carbohydrate-related genes had higher copy numbers in maize than in castor bean, which is reminiscent of the previous findings that key genes involved in oil biosynthesis and turnover have only single copies in castor bean [46]. Strikingly, more lipid- and carbohydrate-related genes were regulated during seed development in castor bean than in maize, and the proportion of regulated lipid- and carbohydrate-related genes was much higher in rapeseed and castor bean than in maize (Table 1 and Fig S1). These findings are in agreement with previous studies that acyl-lipid metabolism genes were differentially expressed between high-oil dicots and low-oil grasses [39]. Hence, we speculated that the lower oil content of maize embryo was not caused by gene family contraction but might owe to the less regulation of lipid- and carbohydrate-related genes.

Although the ratios of LDEG numbers to total DEG numbers were similar among three species, their gene expression patterns and lipid-related pathway enrichment were quite different. Fewer lipid biosynthesis pathways and more lipid metabolism pathways were regulated in maize, and lipid metabolism genes were down-regulated in rapeseed and castor bean but up-regulated in maize (Fig. 3). Therefore, we concluded that more lipid biosynthesis genes and fewer lipid metabolism genes were regulated during lipid accumulation in castor bean endosperm and rapeseed embryo than in maize embryo, potentially leading to the differences in seed oil accumulation between these species.

Broadly speaking, lipid biosynthesis in plants involves FA biosynthesis, TAG assembly, and oil body formation. Genes involved in TAG assembly and lipid-related transcription factor genes were minimally regulated in maize embryo, consistent with the GO enrichment analyses, which indicated that lipid biosynthesis pathways were specifically enriched only in rapeseed and castor bean (Fig. 4 and Data S1). Maize is one of the most important food crops and is increasingly viewed as a potential oil crop because 
of its high levels of polyunsaturated fatty acids $[47,48]$. DGAT gene, whose protein product catalyzes the last step in TAG formation, has been shown to play a vital role in maize seed oil biosynthesis. The oil and oleic acid contents increased by $41 \%$ and $107 \%$ when the high-oil DGAT1-2 allele was ectopically expressed in maize kernels [49]. Likewise, heterologous expression of fungal DGAT2 in maize caused a significant increase in the seed oil content [16]. Thus, we speculated that a lack of DGAT regulation was very likely to limit seed oil biosynthesis in maize. Transcription factors such as LEC1 and WRI1 have been proved to strongly influence seed oil synthesis in maize. Overexpression of $Z m L E C 1$ and $Z m W R / 1$ genes greatly increased the seed oil content of maize kernels and activated target genes that participate in lipid biosynthesis $[23,50]$. Our research indicated that $L E C 1$ was not regulated in maize embryo, and only one out of five WR/1 homologs was differentially expressed in this tissue. Thus, we inferred that the absence of regulation of lipid-related TFs and TAG assembly genes may also limit oil biosynthesis in maize embryo. These genes are therefore promising targets for the improvement of maize seed oil content.

Many carbohydrate-related genes were also differentially regulated in maize embryo. It has been reported that complete or partial glycolytic pathway can provide pyruvate for oil biosynthesis in nonphotosynthetic plants [51]. Although cytosolic glycolysis is commonly regarded as the key carbon metabolism pathway in plants [52], plastidic glycolytic genes such as $P K$ and $P G K$ have also been shown to play important roles in carbon fixation, metabolism, and seed oil biosynthesis in Arabidopsis, rapeseed, and castor bean [28, 53-55]. However, according to our data, most glycolytic genes (e.g., HXK, PFK, PGK, and $P K$ ) were regulated specifically in the cytosol of maize. Moreover, most genes that participate in the PPP and the Calvin Cycle, which could provide substrates and energy for oil biosynthesis, were not regulated in maize embryo (Fig. 6). Thus, we speculated that levels of carbohydrates, which provide substrates for oil biosynthesis, might be limited in the maize embryo due to the absence of regulation of plastidic glycolytic, the PPP, and the Calvin Cycle genes.

Since copy numbers of lipid-related and carbohydrate-related genes were the lowest in castor bean among these species, an interesting question arises: how can castor bean rapidly accumulate so much lipids in seed? In vascular plants, sucrose is the main photoassimilate that moves from sources to sinks for the synthesis of storage compounds, and its metabolism is controlled by two enzymes, INV and SUSy [56]. In our study, only cytosol INV genes (which are minimally active in most plants) were differentially regulated in castor bean endosperm, whereas all castor bean SUSy genes were strongly regulated (Fig. $2 \mathrm{~b}$ and $6 \mathrm{~b}$ ). These findings suggested that castor bean may prefer to utilize fructose for carbon fixation and metabolism. At the same time, lipid biosynthetic genes were highly expressed in castor bean (Fig. 5b), which might account in part for its high seed oil content. Besides, we speculated that castor bean may have evolved some new genes for lipid biosynthesis. This notion is consistent with previous research on the castor bean genome in which it was reported to have evolved an oleic acid hydroxylase gene (FAH) that functions in the biosynthesis of ricinoleic acid [46]. Therefore, it is probable that new genes have been evolved to maintain a high and specific flux of carbohydrates to TAGs in castor bean seeds, a hypothesis that will require experimental verification in the future. 


\section{Conclusions}

Transcriptomic comparisons between rapeseed, castor bean, and maize shed light on the gene regulation profiles of oil biosynthesis in oil storage tissues. The low oil content of maize embryo may have several causes: lower ratio of differentially expressed lipid- and carbohydrate-related genes; less regulation of lipid biosynthesis genes, especially TAG assembly genes and lipid-related TFs, but more regulation of lipid metabolism genes; and the shortage of substrates and energy for oil biosynthesis due to a lack of regulation of the PPP, the Calvin Cycle, and plastidic glycolytic genes. This study provides insight into molecular mechanisms of seed oil accumulation in maize and provides multiple potential targets for improving maize oil production, such as $D G A T, W R / 1, L E C 1, P K$, and $P G K$. These findings improve our understanding of seed oil biosynthesis at the molecular level and provide a solid foundation for further studies, helping to draw a blueprint for the molecular breeding of oilseed crops.

\section{Methods}

\section{Plant growth conditions and sampling.}

The cultivated species of rapeseed (cv. ZS11), castor bean (cv. Youbi No. 4) and maize (cv. Taiyanghua No. 3) were used for analyses. All plants were cultivated at the experimental field with nature growth condition at Wuhan, Hubei, China. Rapeseed seeds were sampled at 15 (S1), 25 (S2), 35 (S3), and 50 (S4) days after flowering, castor bean seeds were sampled at 10 (S1), 20 (S2), 30 (S3), and 40 (S4) days after flowering, and maize seeds were sampled at 15 (S1), 20 (S2), 25 (S3), and 30 (S4) days after flowering according to the seed developmental process of plants. Embryo of rapeseed and maize, and endosperm of castor bean at four stages were separated from the whole seeds for oil content and transcriptome analysis.

\section{Analysis of SOC.}

The rape embryo, castor bean endosperm, and maize embryo (each had six biological replicates) were dried at $37^{\circ} \mathrm{C}$ to eliminate the moisture and the relative oil contents were measured using nuclear magnetic resonance (NMR PQ001; Niumag). The seeds with known oil contents of each species were used to build the baseline for relative oil contents measurement. The detection range of seed weight with NMR PQ001 was about 1.0-1.2 g.

\section{RNA extraction and cDNA synthesis.}

Total RNAs of rapeseed embryo, maize embryo, and castor bean endosperm were extracted for transcriptome analyses. Plant tissues were grounded with liquid nitrogen and total RNAs were isolated following manufacturer's instructions of the RNA extraction kit (9769, TaKaRa). Subsequently, an Illumina TruSeq RNA Sample Prep Kit (Illumina, San Diego, CA, USA) was used to build cDNA libraries.

\section{Transcriptome sequencing and blast searches.}


Generated cDNA libraries were sent to a HiSeq2500-PE125 platform (Illumina) to acquire sequence reads.

The filtered reads were searched against the $B$. napus (ZS11), $R$. communis, and Z. mays (B73) genomes, respectively. Gene expression levels were calculated based on FPKM. The FPKM of S2, S3, and S4 samples were compared to S1 samples to identify the differential expressed genes, and the criterion of fold changes were set to 1 to filter the moderately regulated genes.

\section{Data analysis.}

Gene expression pattern analyses were conducted with the STEM software, and the maximum number of model profiles were set to 50 and the maximum unit changes in model profiles between time points were set to 2. GO term enrichments were run on the RStudio loaded with the GO package, and the false discovery rate was set to 0.05 . Lipid- and carbohydrate-related Arabidopsis genes were cited from the published data [38, 3]. Venn diagram analyses were carried out using an online platform (http:// bioinfogp.cnb.csic.es/tools/venny/).

\section{Abbreviations}

SOC: seed oil content; FA: fatty acid; acetyl-CoA: acetyl-Coenzyme A; ACCase: acetyl-CoA carboxylase; MCMT: malonyl-CoA:ACP malonyltransferase; KAS: $\beta$-ketoacyl-ACP synthase; HAD: hydroxyacyl-ACP dehydrase; ENR: enoyl-ACP reductase; KAR: $\beta$-ketoacyl-ACP reductase; ACP: acyl carrier protein; SAD: stearoyl-ACP desaturase; FAT: acyl-ACP thioesterase; ER: endoplasmic reticulum; TAG: triacylglycerol; GPDHC: glyceraldehyde-3-phosphate dehydrogenase complex; GPAT: glyceral 3-phosphate acyltransferase; LPAAT: 1-lysophosphatidic acid acyltransferase; PAP: phosphatidic acid phosphatase; DGAT: diacylglycerol acyltransferase; PDAT: phospholipid:diacylglycerol acyltransferase; TFs: transcription factors; WRI1: WRINKLED1; LEC1/2: LEAFY COTYLEDON1/2; ABI3: ABSCISIC ACID INSENSITIVE3; FUS3: FUSCA3; PKL: PICKLE; VAL: VP1/ABI3-LIKE; PK: pyruvate kinase; PEPCase: phosphoenolpyruvate carboxylase; OAA: oxaloacetate; PPP: pentose phosphate pathway; GO: Gene Ontology; LDEGs: lipid-related differential expressed genes; CDEGs: carbohydrate-related differential expressed genes; 6PGDH: 6-phosphogluconate dehydrogenase; RPE: ribulose-phosphate 3-epimerase; TK: transketolase; TA: transaldolase; RBCS: ribulose bisphosphate carboxylase; PRK: phosphoribulokinase; HXK: hexokinase; PFK: phosphofructokinase; PGK: phosphoglycerate kinase; INV: invertase; SuSys: sucrose synthase.

\section{Declarations}

\section{Ethics approval and consent to participate}

Not applicable.

\section{Consent for publication}

Not applicable. 
Availability of data and materials

All relevant data can be found within the article and its supporting information. The original sequencing data of transcriptomes are available at the NCBI under BioProjects PRJNA781731, PRJNA781735, and PRJNA781737.

\section{Competing interests}

The authors declare that they have no competing interests.

\section{Funding}

This study was supported from the Agricultural Science and Technology Innovation Program (CAASZDRW202109) and the Central Public-interest Scientific Institution Basal Research Fund (No. 1610172021007).

\section{Author contributions}

$\mathrm{NL}$, and SF performed the experiment; NL, and HL performed the data analysis; NL wrote the manuscript; $\mathrm{JL}, \mathrm{XZ}, \mathrm{MZ}$, and HW contributed to modify the manuscript. All authors read and approved the final manuscript.

\section{Acknowledgements}

Not applicable.

\section{Supporting information}

The online version contains supplementary material.

\section{References}

1. Jaworski J, Cahoon EB. Industrial oils from transgenic plants. Curr Opin Plant Biol. 2003;6(2):17884.

2. Durrett TP, Benning C, Ohlrogge J. Plant triacylglycerols as feedstocks for the production of biofuels. Plant J. 2008;54(4):593-607.

3. Li-Beisson Y, Shorrosh B, Beisson F, Andersson MX, Arondel V, Bates PD et al. Acyl-lipid metabolism. Arabidopsis Book. 2010. p. e0133.

4. Cui YP, Liu ZJ, Zhao YP, Wang YM, Huang Y, Li L et al. Overexpression of Heteromeric GhACCase Subunits Enhanced Oil Accumulation in Upland Cotton. Plant Mol Biol Rep. 2017;35(2):287-97.

5. Voelker TA, Hayes TR, Cranmer AM, Turner JC, Davies HM. Genetic engineering of a quantitative trait: Metabolic and genetic parameters influencing the accumulation of laurate in rapeseed. Plant $\mathrm{J}$. 1996;9(2):229-41. 
6. Roesler K, Shintani D, Savage L, Boddupalli S, Ohlrogge J. Targeting of the Arabidopsis homomeric acetyl-coenzyme A carboxylase to plastids of rapeseeds. Plant physiology. 1997;113(1):75-81.

7. Stoll C, Luhs W, Zarhloul MK, Brummel M, Spener F, Friedt W. Knockout of KASIII regulation changes fatty acid composition in canola (Brassica napus). Eur J Lipid Sci Tech. 2006;108(4):277-86.

8. Wei Q, Li J, Zhang L, Wu PZ, Chen YP, Li MR et al. Cloning and characterization of a beta-ketoacylacyl carrier protein synthase II from Jatropha curcas. J Plant Physiol. 2012;169(8):816-24.

9. Bourgis F, Kilaru A, Cao X, Ngando-Ebongue GF, Drira N, Ohlrogge JB et al. Comparative transcriptome and metabolite analysis of oil palm and date palm mesocarp that differ dramatically in carbon partitioning. P Natl Acad Sci USA. 2011;108(30):12527-32.

10. Salas JJ, Ohlrogge JB. Characterization of substrate specificity of plant FatA and FatB acyl-ACP thioesterases. Arch Biochem Biophys. 2002;403(1):25-34.

11. Shockey J, Regmi A, Cotton K, Adhikari N, Browse J, Bates PD. Identification of Arabidopsis GPAT9 (At5g60620) as an Essential Gene Involved in Triacylglycerol Biosynthesis. Plant physiology. 2016;170(1):163-79.

12. Jain RK, Coffey M, Lai K, Kumar A, MacKenzie SL. Enhancement of seed oil content by expression of glycerol-3-phosphate acyltransferase genes. Biochem Soc T. 2000;28:958-61.

13. Zou JT, Katavic V, Giblin EM, Barton DL, MacKenzie SL, Keller WA et al. Modification of seed oil content and acyl composition in the brassicaceae by expression of a yeast sn-2 acyltransferase gene. Plant Cell. 1997;9(6):909-23.

14. Baud S, Lepiniec L. Physiological and developmental regulation of seed oil production. Progress in Lipid Research. 2010;49(3):235-49.

15. Jako C, Kumar A, Wei YD, Zou JT, Barton DL, Giblin EM et al. Seed-specific over-expression of an Arabidopsis cDNA encoding a diacylglycerol acyltransferase enhances seed oil content and seed weight. Plant physiology. 2001;126(2):861-74.

16. Oakes J, Brackenridge D, Colletti R, Daley M, Hawkins DJ, Xiong H et al. Expression of fungal diacylglycerol acyltransferase2 genes to increase kernel oil in maize. Plant physiology. 2011;155(3):1146-57.

17. Lardizabal K, Effertz R, Levering C, Mai J, Pedroso MC, Jury T et al. Expression of Umbelopsis ramanniana DGAT2A in seed increases oil in soybean. Plant physiology. 2008;148(1):89-96.

18. Dahlqvist A, Stahl U, Lenman $M$, Banas $A$, Lee $M$, Sandager $L$ et al. Phospholipid: diacylglycerol acyltransferase: An enzyme that catalyzes the acyl-CoA-independent formation of triacylglycerol in yeast and plants. P Natl Acad Sci USA. 2000;97(12):6487-92.

19. Stahl U, Carlsson AS, Lenman M, Dahlqvist A, Huang BQ, Banas W et al. Cloning and functional characterization of a Phospholipid: Diacylglycerol acyltransferase from Arabidopsis. Plant physiology. 2004;135(3):1324-35.

20. Siloto RMP, Findlay K, Lopez-Villalobos A, Yeung EC, Nykiforuk CL, Moloney MM. The accumulation of oleosins determines the size of seed oilbodies in Arabidopsis. Plant Cell. 2006;18(8):1961-74. 
21. Grimberg A, Wilkinson M, Snell P, De Vos RP, Gonzalez-Thuillier I, Tawfike A et al. Transitions in wheat endosperm metabolism upon transcriptional induction of oil accumulation by oat endosperm WRINKLED1. Bmc Plant Biol. 2020;20(1):235.

22. Chen B, Zhang G, Li P, Yang J, Guo L, Benning C et al. Multiple GmWRI1s are redundantly involved in seed filling and nodulation by regulating plastidic glycolysis, lipid biosynthesis and hormone signalling in soybean (Glycine max). Plant Biotechnol J. 2020;18(1):155-71.

23. Shen $\mathrm{B}$, Allen WB, Zheng $\mathrm{P}$, Li C, Glassman $\mathrm{K}$, Ranch $\mathrm{J}$ et al. Expression of ZmLEC1 and ZmWR/1 increases seed oil production in maize. Plant physiology. 2010;153(3):980-7.

24. Elahi N, Duncan RW, Stasolla C. Decreased seed oil production in FUSCA3 Brassica napus mutant plants. Plant Physiol Bioch. 2015;96:222-30.

25. Schwender J, Ohlrogge JB. Probing in vivo metabolism by stable isotope labeling of storage lipids and proteins in developing Brassica napus embryos. Plant physiology. 2002;130(1):347-61.

26. Kubis SE, Pike MJ, Hill LM, Rawsthorne S. The import of phosphoenolpyruvate by plastids from developing embryos of oilseed rape, Brassica napus (L.), and its potential as a substrate for fatty acid synthesis. J Exp Bot. 2004;55(402):1455-62.

27. Schwender J, Ohlrogge JB, Shachar-Hill Y. A flux model of glycolysis and the oxidative pentosephosphate pathway in developing Brassica napus embryos. J Biol Chem. 2003;278(32):29442-53.

28. Andre C, Froehlich JE, Moll MR, Benning C. A heteromeric plastidic pyruvate kinase complex involved in seed oil biosynthesis in Arabidopsis. Plant Cell. 2007;19(6):2006-22.

29. Baud S, Wuilleme S, Dubreucq B, de Almeida A, Vuagnat $C$, Lepiniec $L$ et al. Function of plastidial pyruvate kinases in seeds of Arabidopsis thaliana. Plant J. 2007;52(3):405-19.

30. Xu ZP, Li JW, Guo XP, Jin SX, Zhang XL. Metabolic engineering of cottonseed oil biosynthesis pathway via RNA interference. Sci Rep-Uk. 2016;6.

31. Lu L, Wei W, Li QT, Bian XH, Lu X, Hu Y et al. A transcriptional regulatory module controls lipid accumulation in soybean. New Phytol. 2021.

32. Wang LH, Zhang YX, Li DH, Dossa K, Wang ML, Zhou R et al. Gene expression profiles that shape high and low oil content sesames. Bmc Genet. 2019;20.

33. Liu S, Fan CC, Li JN, Cai GQ, Yang QY, Wu J et al. A genome-wide association study reveals novel elite allelic variations in seed oil content of Brassica napus. Theor Appl Genet. 2016;129(6):1203-15.

34. Wang L, Jiang X, Wang L, Wang W, Fu C, Yan X et al. A survey of transcriptome complexity using PacBio single-molecule real-time analysis combined with Illumina RNA sequencing for a better understanding of ricinoleic acid biosynthesis in Ricinus communis. Bmc Genomics. 2019;20(1):456.

35. Cocuron JC, Koubaa M, Kimmelfield R, Ross Z, Alonso AP. A Combined Metabolomics and Fluxomics Analysis Identifies Steps Limiting Oil Synthesis in Maize Embryos. Plant physiology. 2019;181(3):961-75. 
36. Hayden DM, Rolletschek H, Borisjuk L, Corwin J, Kliebenstein DJ, Grimberg A et al. Cofactome analyses reveal enhanced flux of carbon into oil for potential biofuel production. Plant J. 2011;67(6):1018-28.

37. Guerin C, Joet T, Serret J, Lashermes P, Vaissayre V, Agbessi MD et al. Gene coexpression network analysis of oil biosynthesis in an interspecific backcross of oil palm. Plant J. 2016;87(5):423-41.

38. Troncoso-Ponce MA, Kilaru A, Cao X, Durrett TP, Fan J, Jensen JK et al. Comparative deep transcriptional profiling of four developing oilseeds. Plant J. 2011;68(6):1014-27.

39. Zhang L, Wang SB, Li QG, Song J, Hao YQ, Zhou L et al. An integrated bioinformatics analysis reveals divergent evolutionary pattern of oil biosynthesis in high- and low-oil plants. PloS one. 2016;11(5):e0154882.

40. Zhang Z, Dunwell JM, Zhang YM. An integrated omics analysis reveals molecular mechanisms that are associated with differences in seed oil content between Glycine max and Brassica napus. Bmc Plant Biol. 2018;18(1):328.

41. Zhou H, Xia D, Li P, Ao Y, Xu X, Wan S et al. Genetic architecture and key genes controlling the diversity of oil composition in rice grains. Molecular plant. 2020.

42. Li H, Peng Z, Yang X, Wang W, Fu J, Wang J et al. Genome-wide association study dissects the genetic architecture of oil biosynthesis in maize kernels. Nature genetics. 2013;45(1):43-50.

43. Cagliari A, Margis-Pinheiro M, Loss G, Mastroberti AA, de Araujo Mariath JE, Margis R. Identification and expression analysis of castor bean (Ricinus communis) genes encoding enzymes from the triacylglycerol biosynthesis pathway. Plant Sci. 2010;179(5):499-509.

44. Amiruddin N, Chan PL, Azizi N, Morris PE, Chan KL, Ong PW et al. Characterization of Oil Palm AcylCoA-Binding Proteins and Correlation of Their Gene Expression with Oil Synthesis. Plant Cell Physiol. 2020;61(4):735-47.

45. Chalhoub B, Denoeud F, Liu SY, Parkin IAP, Tang HB, Wang XY et al. Early allopolyploid evolution in the post-Neolithic Brassica napus oilseed genome. Science. 2014;345(6199):950-3.

46. Chan AP, Crabtree J, Zhao Q, Lorenzi H, Orvis J, Puiu D et al. Draft genome sequence of the oilseed species Ricinus communis. Nat Biotechnol. 2010;28(9):951-U3.

47. Godfray HCJ, Beddington JR, Crute IR, Haddad L, Lawrence D, Muir JF et al. Food Security: The Challenge of Feeding 9 Billion People. Science. 2010;327(5967):812-8.

48. Chen J, Zeng B, Zhang M, Xie SJ, Wang GK, Hauck A et al. Dynamic Transcriptome Landscape of Maize Embryo and Endosperm Development. Plant physiology. 2014;166(1):252-64.

49. Zheng P, Allen WB, Roesler K, Williams ME, Zhang S, Li J et al. A phenylalanine in DGAT is a key determinant of oil content and composition in maize. Nature genetics. 2008;40(3):367-72.

50. Pouvreau B, Baud S, Vernoud V, Morin V, Py C, Gendrot G et al. Duplicate maize Wrinkled1 transcription factors activate target genes involved in seed oil biosynthesis. Plant physiology. 2011;156(2):674-86. 
51. Neuhaus HE, Emes MJ. Nonphotosynthetic metabolism in plastids. Annu Rev Plant Phys. 2000;51:111-40.

52. Rawsthorne S. Carbon flux and fatty acid synthesis in plants. Progress in Lipid Research. 2002;41(2):182-96.

53. Li RZ, Qiu ZM, Wang XG, Gong PP, Xu QZ, Yu QB et al. Pooled CRISPR/Cas9 reveals redundant roles of plastidial phosphoglycerate kinases in carbon fixation and metabolism. Plant $\mathrm{J}$. 2019;98(6):1078-89.

54. White JA, Todd T, Newman T, Focks N, Girke T, de llarduya OM et al. A new set of Arabidopsis expressed sequence tags from developing seeds. The metabolic pathway from carbohydrates to seed oil. Plant physiology. 2000;124(4):1582-94.

55. Simcox PD, Reid EE, Canvin DT, Dennis DT. Enzymes of the Glycolytic and Pentose Phosphate Pathways in Proplastids from the Developing Endosperm of Ricinus communis L. Plant physiology. 1977;59(6):1128-32.

56. Koch K. Sucrose metabolism: regulatory mechanisms and pivotal roles in sugar sensing and plant development. Curr Opin Plant Biol. 2004;7(3):235-46.

\section{Figures}



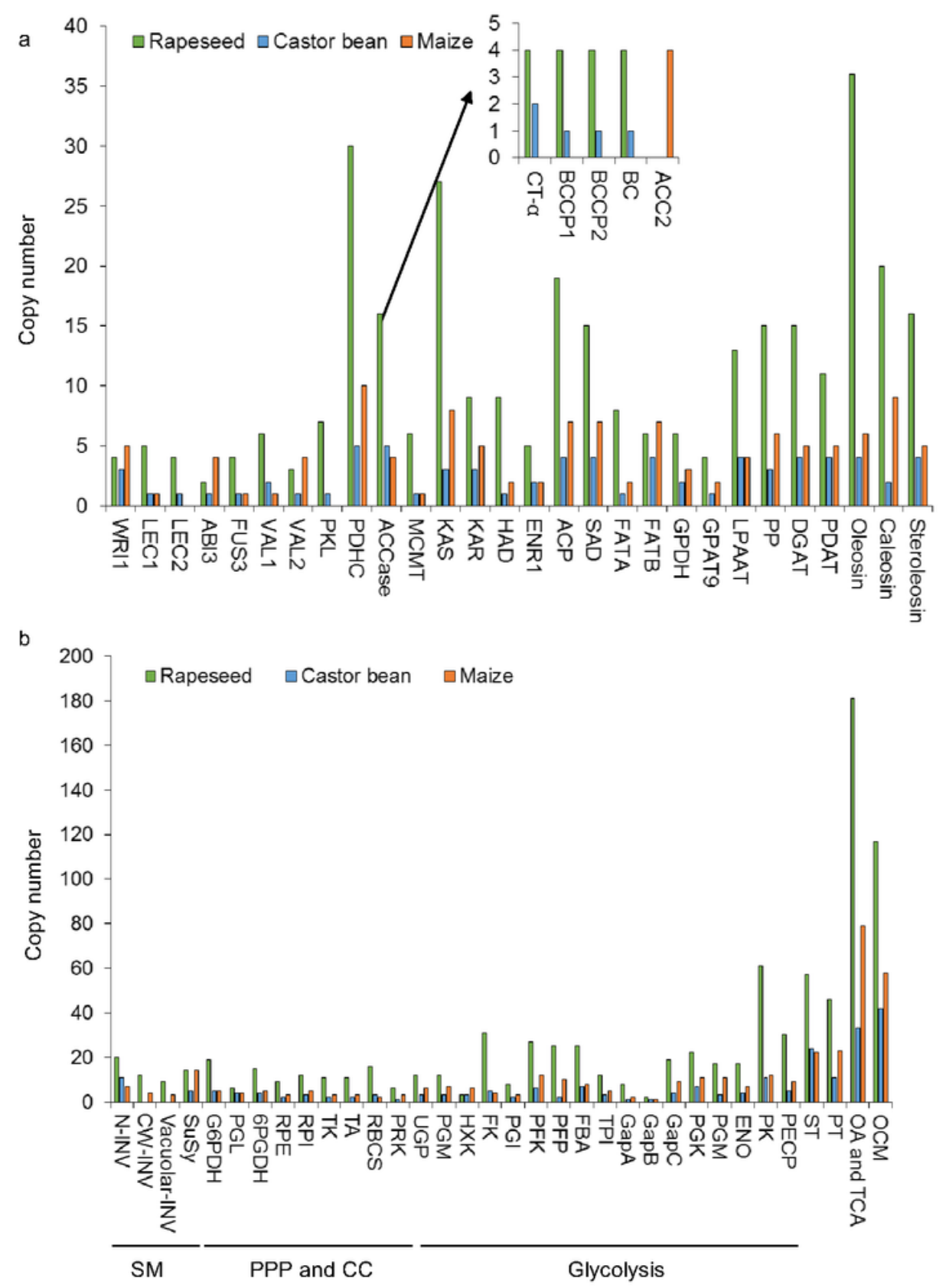

\section{Figure 1}

Relative copy number variation analyses of lipid- and carbohydrate-related genes in three species. a, lipidrelated genes including FA biosynthesis, TAG assembly, oil body formation, and lipid-related TFs. a, plastidial fatty acid synthesis; b, TAG synthesis; c, plastidial glycerolipid, galactolipid and sulfolipid synthesis; d, eukaryotic phospholipid synthesis; e, beta-oxidation; f, TAG degradation; g, mitochondrial fatty acid and lipoic acid synthesis; h, mitochondrial phospholipid synthesis; i, lipid trafficking; j, 
sphingolipid synthesis; $\mathrm{k}$, lipid signaling; I, miscellaneous: lipid related; $m$, fatty acid elongation and cuticular wax synthesis; $n$, cutin synthesis; 0 , cuticular wax synthesis; $p$, aliphatic suberin synthesis; $q$, aromatic suberin synthesis; r, phospholipase; s, lipid acylhydrolase; t, GDSL; u, galactolipid degradation; v, lipase. b, carbohydrate-related genes that participated in different carbon metabolism pathways. SM, sucrose metabolism; PPP and CC, pentose phosphate pathway and calvin cycle; ST, sugar transporters; PT, plastid transporters; OA and TCA, organic acid and TCA; OCA, other carbohydrate metabolism.

a

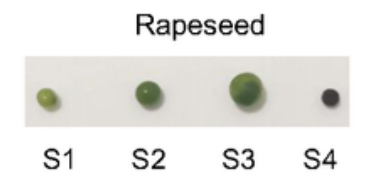

Rapeseed embryo

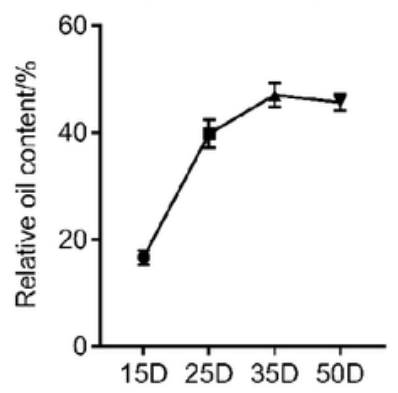

Castor bean

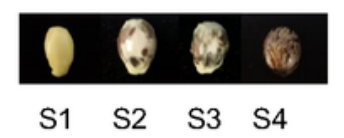

Castor bean endosperm

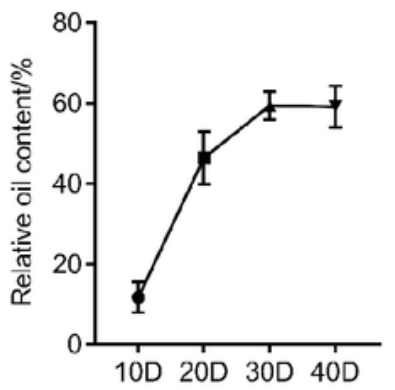

Maize

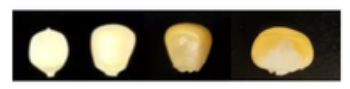

S1 $\quad$ S2 $\quad$ S3 $\quad$ S4

Maize embryo

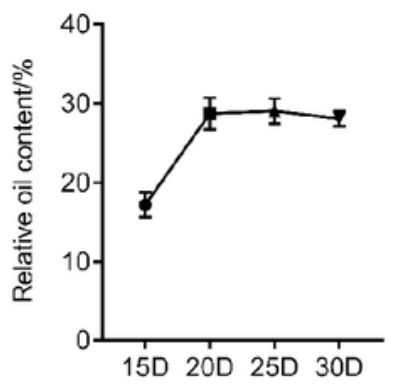

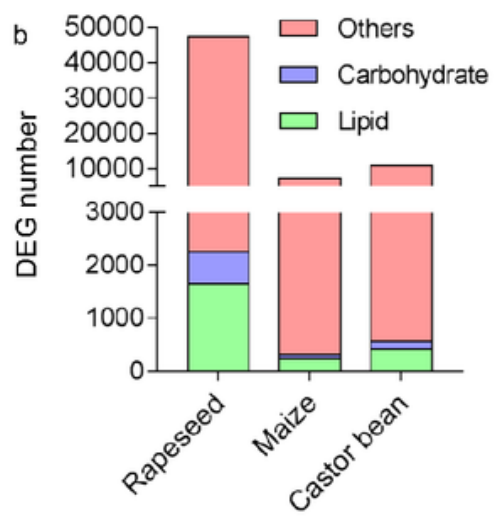

c

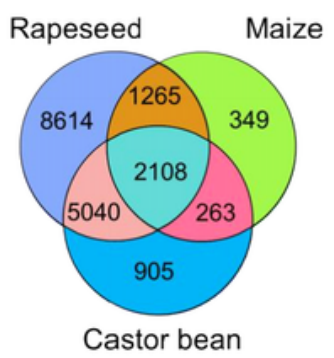

Figure 2 
Transcriptome analyses of rapeseed, castor bean, and maize during the seed development. a, the seed phenotypes and seed oil content of rapeseed embryo, castor bean endosperm, and maize embryo at four developmental stages (S1-S4). b, numbers differential expressed gene (DEG) of rapeseed, castor bean, and maize during seed development. c, venn diagrams of DEGs in three species, all genes were compared to Arabidopsis to find the homologous genes.

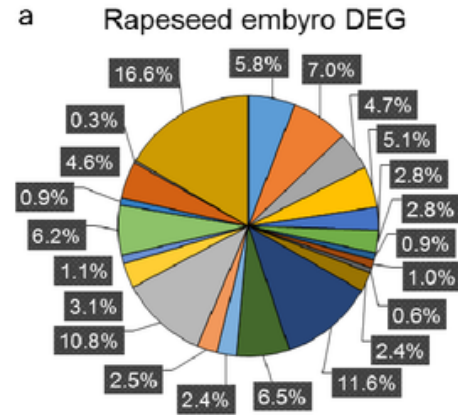

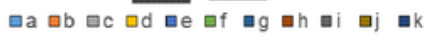
घ) घm घn घ० घp घq ar घs घt घu घv

b

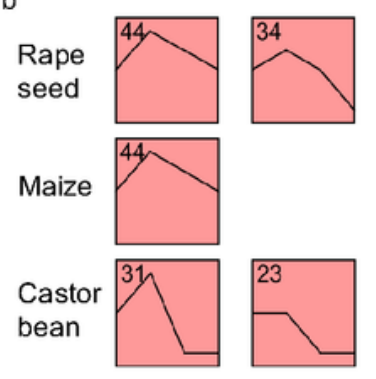

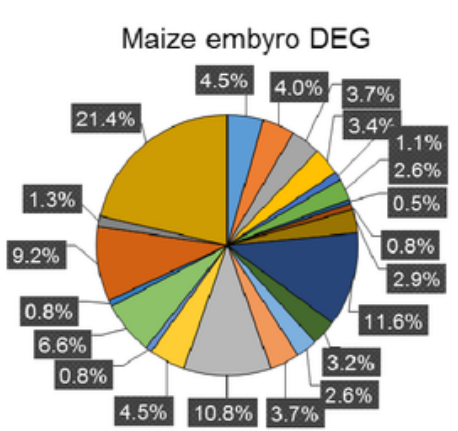

घa घb घc घd घe घf घg घh घi घj घk

घ am घn घ० वp घq घr घs घt घu घv

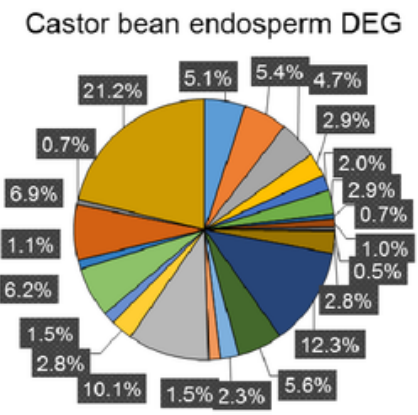

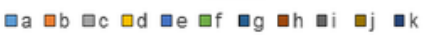

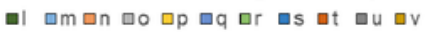

c
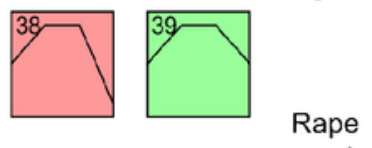

seed
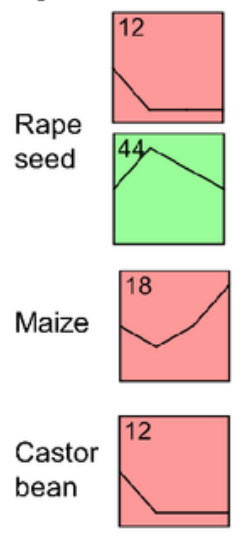
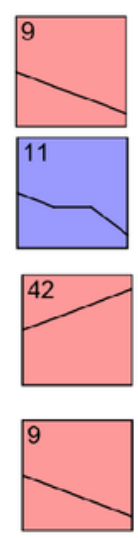
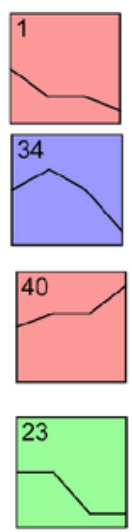
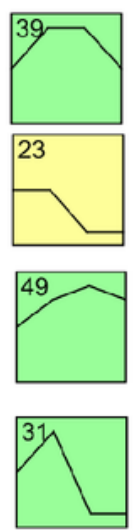

Figure 3 
Lipid metabolism pathways and the gene expression patterns of LDEGs of rapeseed, castor bean, and maize. a, the proportion of rapeseed, castor bean, and maize LDEGs in different lipid metabolism categories. b and c, the expression pattern clusters of lipid biosynthetic (b) and lipid metabolic (c) DEGs in three species.
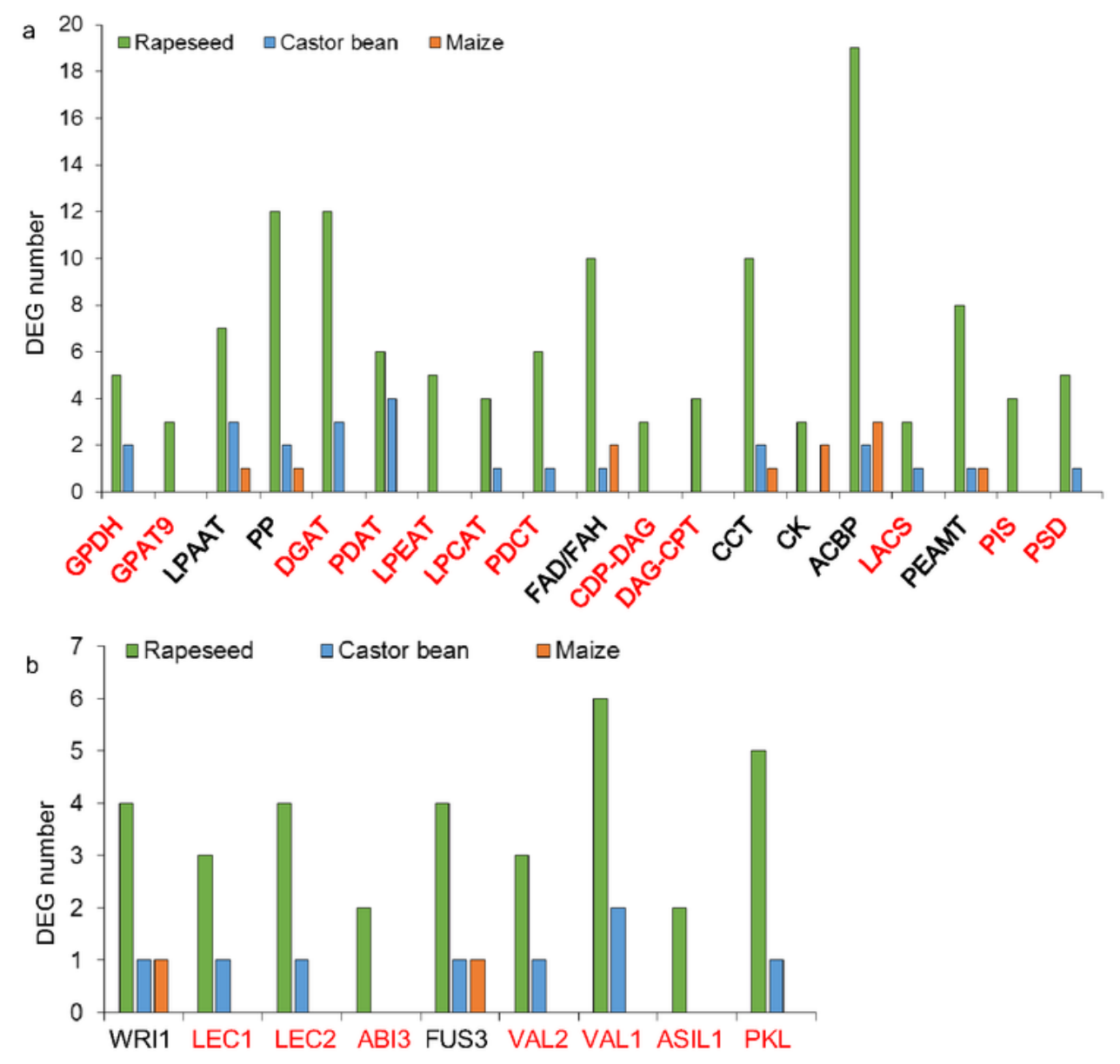

Figure 4 
Numbers of differential expressed genes that participated in TAG biosynthesis (a) and vital lipid-related TFs (b) of rapeseed, castor bean, and maize during seed development.

a

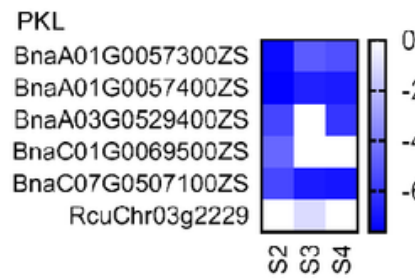

VAL2

BnaA03G0530000ZS

BnaA08G0071600ZS

BnaC07G0507700ZS

RcuChr10g0704

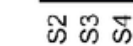

b

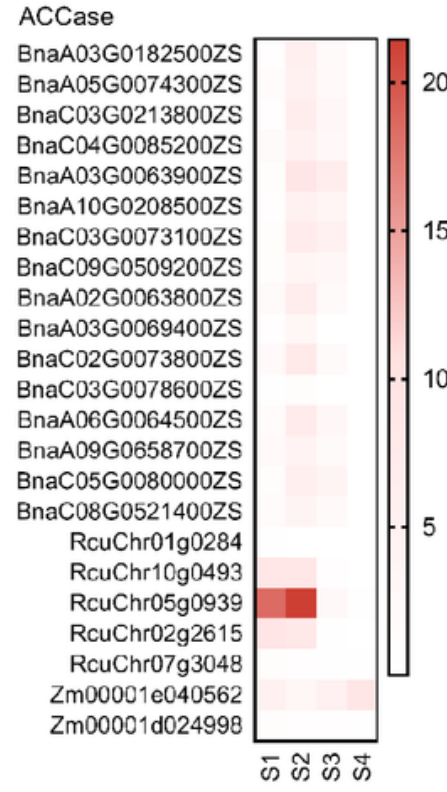

ACCase

BnaA03G0182500ZS

BnaA05G0074300ZS

BnaC03G0213800ZS

BnaC04G0085200ZS

BnaA03G0063900ZS

BnaA10G0208500ZS BnaC03G0073100ZS

BnaC09G0509200ZS

BnaA02G0063800ZS

BnaA03G0069400ZS

BnaC02G0073800Zs

BnaC03G0078600ZS

4 BnaA06G0064500ZS

BnaA09G0658700ZS

BnaC05G0080000ZS

BnaC08G0521400ZS

RcuChr01g0284

RcuChr10g0493

RcuChr05g0939

RcuChr02g2615

Zm00001e040562

\section{ஸूฆั}

DGAT

BnaA07G0011800ZS

BnaA09G0121200ZS

BnaC04G0279500ZS

BnaC07G0026200ZS

BnaC09G0126800ZS

15 BnaC09G0541200ZS

BnaA01G0206700ZS

BnaA03G0420700ZS

BnaC01G0259700ZS

BnaC07G0393500ZS

BnaA10G0113700ZS

BnaC09G0376500ZS

RcuChr03g1635

RcuChr05g0358

RcuChr09g1002

$\mathrm{Zm00001d037760}$

Zm00001d051628

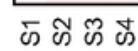

0

$-5$

4

2

6

0

(1)

\section{Figure 5}

The regulation of lipid biosynthetic genes during the seed development between three oilseeds. a, the fold changes of genes that encoding ACCase and two lipid-related TFs: PKL and VAL2. b, the relative gene 
expression levels of some lipid biosynthesis genes: ACCase and DGAT. To evaluate the relative gene expression levels, the FPKMs were used for the calculation.

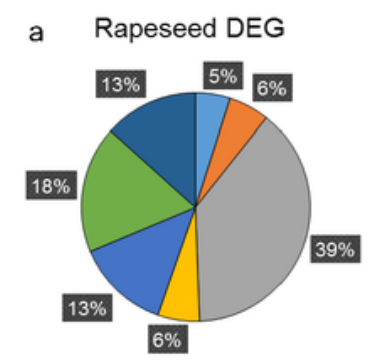

口Sucrose Metabolism

$\square$ Plastid Transporters

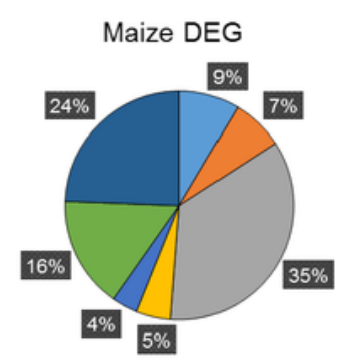

ロSugar Transporters

口PPP and Calvin Cycle

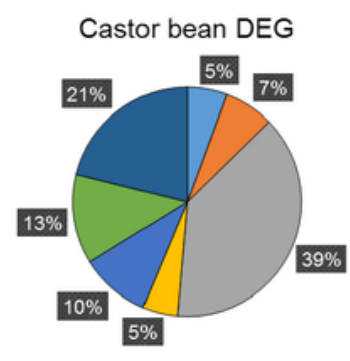

$\square$ Glycolysis

$\square$ Organic Acid and TCA

nOther Carbohvdrate Metabolism

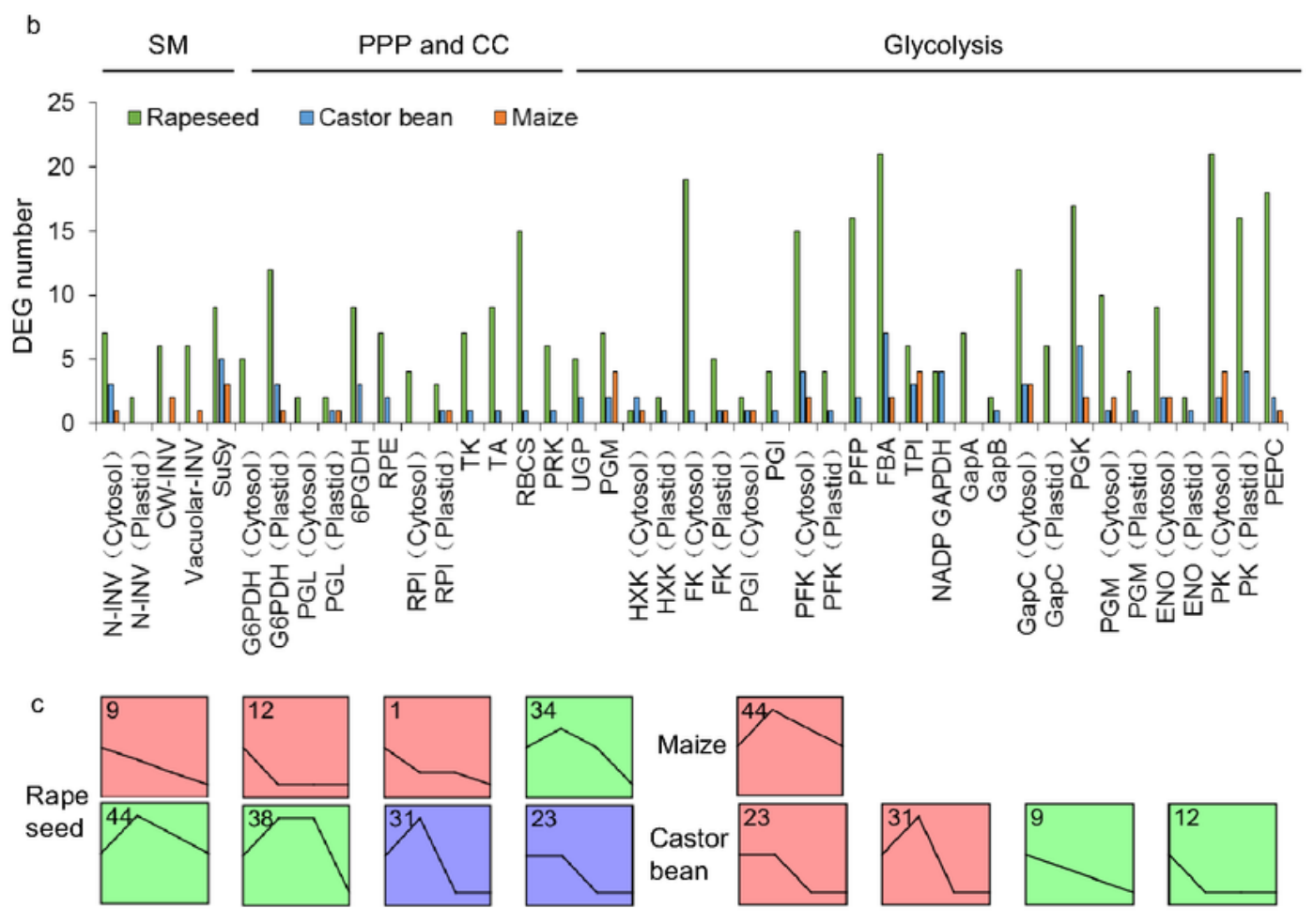

\section{Figure 6}

Differential regulation of CDEGs between three species. a, pie chart showed the proportion of rapeseed, castor bean, and maize CDEGs in different carbon metabolism categories. $b$, numbers of differential expressed genes that participated in different carbohydrate metabolism pathways such as sucrose 
metabolism (SM), glycolysis, pentose phosphate pathway and Calvin Cycle (PPP and CC) of rapeseed, castor bean, and maize during seed development. c, the expression pattern clusters of carbohydraterelated genes in three plants.

\section{Supplementary Files}

This is a list of supplementary files associated with this preprint. Click to download.

- DataS1.xlsx

- SupplementalFigures15.pdf 